\title{
Distance based Routing Scheme for Underwater Sensor Networks
}

\author{
Abraham Varughese, \\ NSTL \\ Visakahaptnam \\ India
Rangarajan S.V
NSTL
Visakahaptnam
India

\author{
Seetharamaiah.P \\ Andhra University \\ Visakahaptnam \\ India
Soundararajan.K
JNTU
Anantapur
India

\begin{abstract}
Underwater Sensor Networks (UWSN) are gaining increasing importance. The main constituents of UWSN are Static Acoustic sensor nodes and Autonomous Underwater Vehicles. The sensor nodes located in a particular depth are to pass the data to the sinks. As acoustic transmission range is limited, the information has to be routed via many hops. We are proposing a distance based routing scheme for such an under water network. The performance of the network employing a distance based routing scheme has been evaluated for different scenarios using UNET underwater network simulator.
\end{abstract}

\section{General Terms}

Network Simulation

\section{Keywords}

Simulation, Acoustic, Network, AUV, Embedded, Protocols, Routing

\section{INTRODUCTION}

Man in his quest for knowledge has forayed much into his habitat, yet so much area on the blue planet remains unexplored. He has a lot of knowledge, but much is left to be understood. With more than $70 \%$ of the earth covered by water, significant research efforts are needed to familiarize ourselves with the mysteries of the unfathomable depths of the huge water bodies.

Developments in communication and computers provided the necessary impetus to the networking of computer systems which revolutionised the information sharing and spread of knowledge. Advent of VLSI technology has made the miniaturisation of electronic circuits possible. Advancements in sensors and solid state physics has led to the MEMs being used in the measurement of parameters of physical systems. Sensor Networks has emerged as an amalgamation of these two areas namely sensors and communication systems. The communication backbone may be either wired or wireless. The applications of wireless Sensor Networks are vivid and varied. It has revolutionised the area of measurement and networking. Meanwhile man has started exploring the depths of oceans for natural resources.
Last few years witnessed the individual and combined efforts of acoustics and networking in the realm of underwater water networks. Underwater networking has become a new research area due to its immense applications. Underwater networking is rather unexplored area. Unlike their terrestrial counterparts, there are no standards available for underwater sensor networks. As electromagnetic waves do not propagate well underwater, acoustics plays a key role in underwater communication. Due to considerable differences in the distinctiveness of electromagnetic and acoustic channels, networking protocols for underwater systems differ from those developed for wired and wireless radio networks.

There are wide and varied applications for Underwater Sensor Networks. These include study of ocean resources, protection of installations at the sea coast, monitoring of enemy intrusions and study of flora and fauna in sea. As suggested in [1] the constituents of Underwater Sensor Networks can be static sensor nodes, and Autonomous Underwater vehicles. We had proposed in [2] that hovering sensor nodes also can be a useful constituent in UWSN.

The main purpose of the network of nodes is to pass the requisite information from a sensor node to a central station. Before deploying an underwater network, it will be prudent to have a simulation of the working of the nodes and their throughput. This paper discusses a distance based routing scheme for an underwater network. Further a program has been developed in Visual Basic to simulate the nodes and data transfer in underwater water sensor network. The performance of the routing scheme was analyzed using this simulator.

\section{UNDERWATER ACOUSTIC NETWORK -DESIGN CHALLENGES}

Designing, commissioning and deploying an Underwater Sensor Network has got varied challenges. The on board electronics has to be essentially low power consuming. Ruggedness and reliability of the electronics is another important factor. The mechanical housing design of an underwater node is also complex as it has to withstand the pressure of water at depths[5].

Deploying an underwater acoustic network is a time consuming task as platforms are to sail in the conducive 
climatic conditions. The paraphernalia required for such a deployment is huge. This is in contrast to the situation on land. In addition to this, the deployed sensors are susceptible to corrosion.

There are no established standards available for UWSN. The hardware and software interfaces are still not clearly defined. No uniformity exists over the networking protocols. [4].

The communication in underwater is also a difficult proposition because of the restriction in the medium. The attenuation of electro magnetic waves in underwater is another limiting factor. Hence acoustic communication is the only possible method of communication. Speed of acoustic signal is $1500 \mathrm{~m} / \mathrm{s}$ and hence the propagation delay of acoustic waves is quite high compared electromagnetic signal in air. Also the acoustic signal gets attenuated due to spreading, absorption and scattering [6].

\section{NETWORK ARCHITECTURE}

The underwater sensor nodes can be of three types namely static sensor nodes, hovering sensor nodes and Autonomous Underwater Vehicles.

Static nodes will be either anchored to the sea bottom or it can be a floating node. The node can be floating based on its positive buoyancy or an auxiliary device can be used to make it float. These nodes have got acoustic modems to communicate between each other. These nodes are equipped with GPS receiver and satellite communication device. Static sensor node will have a central controller based on a low power micro controller/ FPGA. Central controller will function using the on board battery. Controller interacts with the sensors and with the acoustic modem. Controller monitors the data using its on board sensors. The electronics and sensors are housed in a mechanical console which is water proof.

We have proposed in [7] sensor nodes which can hover. Sensor nodes will be having hovering facility as option. These nodes will have almost neutral buoyancy. Hence they will be able to stay at the fixed depths in the sea, if the effects of currents are less. These nodes will be able to operate the small onboard motor for changing the depth or to achieve hovering action.

Autonomous underwater vehicles are unmanned autonomous self propelled vehicles which can be set to pre programmed trajectories with autopilot. These autonomous vehicles can accomplish specific missions either for civilian or military applications. Based on their displacement AUVs can be classified as heavy weight or light weight.

The localization of these underwater nodes/vehicles is another merging area [9]. We had suggested in [11], how an hovering node can pass the location information by coming to the surface.

\subsection{Routing Schemes}

The network layer of the protocol has got the responsibility of finding out the path between a source sensor node to the destination node which is generally the sink. Several routing schemes have been proposed in the literature [9]. The existing routing protocols comes under three classifications namely Proactive protocols, reactive protocols and geographical protocols. Proactive protocols keeps up-to-date routing information from each node to other nodes. In the case of Reactive protocols, a node initiates a route discovery process only when a route to a destination is required. Geographical routing protocols make use of the localization information to find out the path from source to destination [1].

It is desirable to have a routing strategy which results in lesser traffic and less energy consumption. In order to realize such a protocol some optimizations are to be carried out. One way of realizing the optimization can be in the form of lesser number of retransmissions[7]. This can be achieved only if the message follows the shortest path. In order to calculate the shortest path, it is required to have multiple transmissions and receptions. Depth based Algorithm which will reduce the number of transmissions has been suggested in [12]. We are proposing here a Distance based Algorithm which makes use of the knowledge of the distance to the sink.

\subsection{Protocol Overview}

The protocol is based on the distance of the node from the sink. This algorithm tries to deliver the packet to the sink through minimum number of nodes. If we deliver the packet through nodes which are at decreasing distance from the sink, it is likely that the message traverses through lesser number of nodes. It is possible that all the receiving nodes of packet can retransmit the data. Upon receiving a packet, the node examines whether the distance of the node from the sink is less than the distance of the sender from the sink. If it is less, the packet is forwarded to the next node. It is assumed that all the nodes are having the distance information to the sink. This can be achieved by periodically sending a Distancefind signal from the sink. This signal will be analog acoustic signal of known amplitude. Each node, on receiving the signal, will calculate the Received Signal Strength (RSSI). Based on the RSSI, the distance field of the node will be updated. Incase a node does not receive the Distancefind signal, the distance field will remain at maximum value.

\subsubsection{Reduction of redundant packets}

If multiple paths are employed to forward the packets, it leads to redundant traffic. It is evident that when same packets are transmitted multiple times, it leads to energy drain and increased traffic. This is avoided by using the Unique id field of the message. Further the "Previous transmitted node" info is used to avoid any looping of messages back and forth between the nodes. When a data packet is received at any node and the packet is examined to see whether this particular packet has been forwarded by the node early. In case the particular packet has been forwarded by the node early, then the packet is dropped. Secondly, some more packets are dropped by the distance consideration. On receiving a packet, node decides whether it is a qualified forwarder by examining the distance info.

\section{PERFORMANCE EVALUATION}

The performance of the sensor network has been carried out using the UNET simulator. We have explained the development of UNET, a simulation platform for underwater networks in [13]. 


\subsection{UNET simulator}

Unlike in a terrestrial network, the underwater network is in a three dimensional space. The $\mathrm{x}, \mathrm{y}$ and $\mathrm{z}$ coordinates of the node are to be considered. In a terrestrial network generally the $\mathrm{z}$ coordinate is not of significance as the height from the surface is of lesser significance in terrestrial scenario. However the depth at which the node is placed is of paramount importance in an under water scenarioError! Reference source not found.. UNET simulates the routing of packets across different nodes. The input to UNET simulator is indicated in table 1 .

Table 1: Input parameters to the UNET

\begin{tabular}{|l|l|}
\hline Input Parameter & Parameter Value \\
\hline Simulation Time & Programmable \\
\hline Area of interest & $10 \mathrm{~km}$ x 10 km \\
\hline $\begin{array}{l}\text { The Range of the } \\
\text { Acoustic Link }\end{array}$ & $3000 \mathrm{~m}$ \\
\hline $\begin{array}{l}\text { Velocity of the sound in } \\
\text { water }\end{array}$ & $1500 \mathrm{~m} / \mathrm{s}$ \\
\hline Total number of nodes & 20 \\
\hline $\begin{array}{l}\text { Maximum number of } \\
\text { hopes (max_hop ) }\end{array}$ & 3 \\
\hline Data Interval & Programmable \\
\hline Data Duration & In milli seconds \\
\hline Routing Protocol & Can be programmed \\
\hline Node Placement Model & Random/fixed \\
\hline Antenna Type & Omni Directional \\
\hline Node mobility & Programmable \\
\hline Data Transfer & CBR \\
\hline
\end{tabular}

The message has got a header which will used to know the start of the message. The message contains a unique id which will be generated in the source node. The message also will have the information of the source node and destination node. Whenever the data is ready for transmission by the higher layer, transmitter buffer is updated with the data. Once the data is present in the transmitter buffer, the medium layer carries out the propagation of the data. Data will be received in the nodes based on the distance from the source and time of travel.

The message format is illustrated in figure 2. It consists of Packet header, The control bytes, message identification number, distance info and the trailer. Message identification number will be used to identify different messages. Distance info will be updated hop by hop when it traverses each node.
Table 2 Message Structure

\begin{tabular}{|l|l|}
\hline 0 & Packet Header \\
\hline 1 & Control Bytes \\
\hline 2 & Unique Id \\
\hline 3 & Message Id \\
\hline 4 & Source \\
\hline 5 & Destination \\
\hline 6 & Current Source \\
\hline 7 & Hope count \\
\hline 8 & Trailer \\
\hline
\end{tabular}

Each node has got a data structure associated with it. This data structure stores the data corresponding to the node. Whenever a message is received at the node / transmitted from the node, the data structure is updated. The transmitter buffer and receiver buffer will hold the data which is to be transmitted or received.

Table 3 : Node Data Structure

\begin{tabular}{|l|l|}
\hline 0 & Tx Ready \\
\hline 1 & Rx Ready \\
\hline 2 & Message Id \\
\hline 3 & Destn \\
\hline 4 & Unique Id \\
\hline 5 & Energy \\
\hline 6 & No of Tx \\
\hline 7 & No of rx \\
\hline
\end{tabular}

Table 4:Output Parameters

\begin{tabular}{|c|}
\hline Description \\
\hline Id of the message \\
\hline $\begin{array}{l}\text { This parameter represents the total } \\
\text { number of data packets that. }\end{array}$ \\
\hline $\begin{array}{l}\text { Number of packets rejected because } \\
\text { destination is busy }\end{array}$ \\
\hline $\begin{array}{l}\text { Number of packets rejected because the } \\
\text { hope count is more. }\end{array}$ \\
\hline $\begin{array}{l}\text { Number of packets rejected because the } \\
\text { packets are have reached the same node } \\
\text { again. }\end{array}$ \\
\hline $\begin{array}{l}\text { Packets which are transmitted from a } \\
\text { node since destination is a different node }\end{array}$ \\
\hline $\begin{array}{l}\text { No of packets which successfully reached } \\
\text { the network's sink / destination node }\end{array}$ \\
\hline
\end{tabular}




\subsection{Simulation Scenario}

The position of the nodes has been carried out in a required area of $5 \mathrm{~km} \times 5 \mathrm{~km} \times 4 \mathrm{~km}$. The position of individual node has been fixed in a random way. It is initially assumed that each node does not know the location of the other nodes. Each node is designated by the $\mathrm{x}, \mathrm{y}$ and $\mathrm{z}$ coordinates. An example indicating the placement of 15 nodes which has been randomly generated is shown in figure.

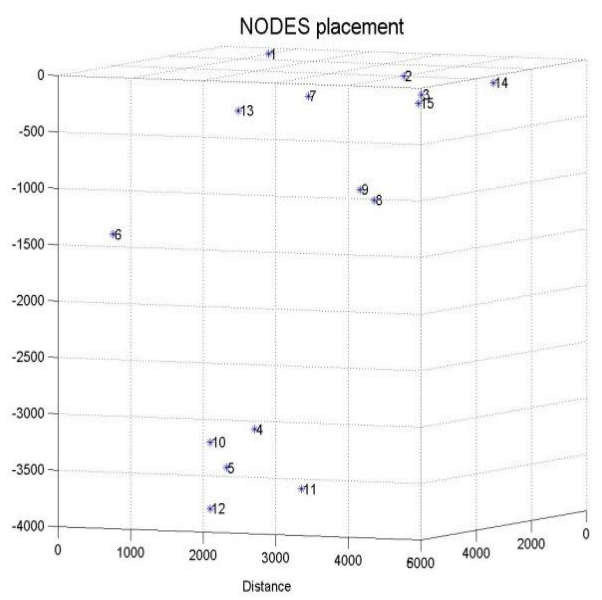

Figure 1: Placement of Nodes

The nodes which has been randomly generated has been pictorially placed in the Error! Reference source not found. Sinks are deployed on the water surface. Nodes are randomly picked for transmission of data.

\subsubsection{Scenario 1}

We have considered a scenario where number of nodes are 50. The total time during which messages will be generated is $30 \mathrm{sec}$. During 30 seconds, 20 messages have been initiated in this network. We have simulated the scenario in UNET, the results are shown in table 5.

Table 5: Scenario 1 Output

\begin{tabular}{|c|c|c|c|}
\hline \multicolumn{3}{|l|}{ Nodes } & $\mathbf{5 0}$ \\
\hline \multicolumn{3}{|c|}{ Number of messages } & 20 \\
\hline \multicolumn{3}{|l|}{ Total time } & 30 \\
\hline & $\begin{array}{l}\text { No } \\
\text { algorit } \\
\mathrm{hm}\end{array}$ & DBF & $\begin{array}{l}\text { Distan } \\
\text { ce } \\
\text { based } \\
\text { algo }\end{array}$ \\
\hline Tx Nodes & 328 & 135 & 99 \\
\hline Tx messages & 16072 & 6615 & 4851 \\
\hline Nodes in Range & 1680 & 725 & 623 \\
\hline
\end{tabular}

\begin{tabular}{|c|c|c|c|}
\hline Nodes busy & 983 & 350 & 269 \\
\hline $\begin{array}{l}\text { Messages } \\
\text { Reached the } \\
\text { next node }\end{array}$ & 697 & 375 & 354 \\
\hline $\begin{array}{l}\text { Retxed } \\
\text { meassges }\end{array}$ & 308 & 115 & 79 \\
\hline $\begin{array}{l}\text { Messages } \\
\text { rejected Hop } \\
\text { count more }\end{array}$ & 055 & 4 & 6 \\
\hline Rejected by algo & & 88 & 120 \\
\hline $\begin{array}{l}\text { Messages } \\
\text { rejected } \\
\text { Repeated msg }\end{array}$ & 324 & 161 & 141 \\
\hline $\begin{array}{l}\text { Messages } \\
\text { reached destn }\end{array}$ & 010 & 7 & 8 \\
\hline
\end{tabular}

Figure 2: Scenario 1 Output

\subsubsection{Scenario 2}

We have considered a scenario where number of nodes are 50. During 50 seconds, 40 messages have been initiated in this network. We have simulated the scenario in UNET, and results are illustrated in the graphs.

Table 6

\begin{tabular}{|l|l|l|}
\hline Nodes & $:$ & 50 \\
\hline $\begin{array}{l}\text { Number of } \\
\text { messages }\end{array}$ & $:$ & 40 \\
\hline Total time & $:$ & 50 \\
\hline
\end{tabular}

The total number of nodes which attempted for transmission are tabulated. Out of the total transmissions, some of them will not reach the next node as the node is not within the transmission range of the first node. Few more will rejected as the channel is busy.

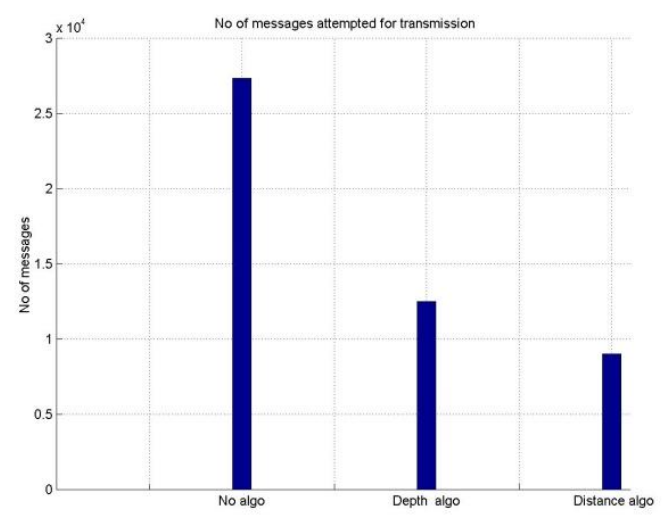

Figure 3: No of messages attempted for $\mathrm{Tx}$ 


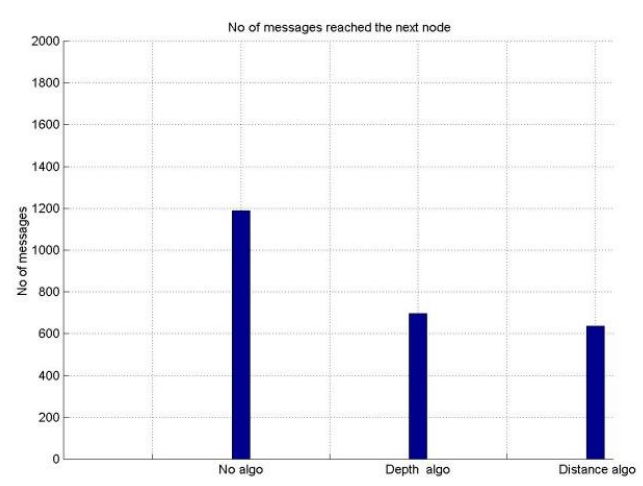

Figure 4 : Messages reached the next node

Messages finally delivered to the destination are indicated in Figure 5. This parameter gives the total number of packets which has reached the sink of the network

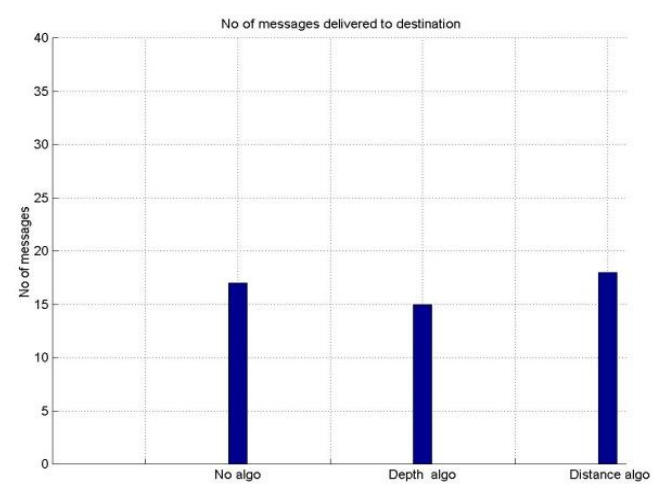

Figure 5: Messages Delivered

The average residual energy in all the nodes are indicated in figure 6.

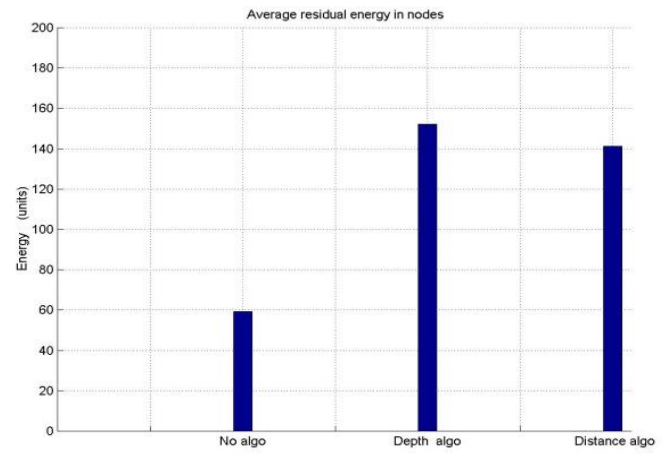

Figure 6 : Residual energy

From the results, it is clear that the distance based algorithm has reduced the number of transmissions in the network.

\section{CONCLUSION}

Realizing an underwater acoustic network, is a difficult task, This paper has dealt about the comparison of routing strategies for such a network. We have proposed a distance based routing scheme which was evaluated using the UNET simulator. We have shown that the number of transmissions can minimized by using this scheme. Further it has been shown that there is a considerable saving of energy. As realizing physical network is a costly process, UNET simulator helps in reviewing the routing strategies in a network.

\section{REFERENCES}

[1] I.F.Akyiditz, D. Pompli and T.Melodia, "Underwater sensor networks: Research challenges", Adhoc Networks, 2005, Elsevier.B.V,3(3):257-279, May 2005.

[2] S.V.Rangarajan, Abraham Varughese, P.Seetharamiah, K.Soundararajan, "Embedded Systems and Sensors for an Acoustic Sensor Network", International Conference on Power, Control and Embedded Systems, ICPCES-2010.

[3] Zaihan Jiang, "Underwater Acoustic Networks: Issues and solutions", International Journal of Intelligent Control and Systems, Vol 13, No.3, September 2008, 152-161.

[4] Mandar Chitre, Lee Freitag, Ethnem Sozer, Shiraz Shahabudeen, Milica Stojanovic and John Potter, "An Architecture for Underwater Networks", Oceans 06.

[5] Jim Partan, Jim Kurose and Brian Niel Nevine , "A Survey of Practical Issues in Underwater Networks", WUVNet 06, September, 2006, Los angels, USA.

[6] R.J.Urick, Principles of Underwater Sound, McGraw-Hill, Newyork, 1961.

[7] Ayaz M, et al. A survey on routing techniques in underwater wireless sensor networks. J Network Comput Appl (2011), doi:10.1016/j.jnca.2011.06.009

[8] S.V.Rangarajan, Abraham Varughese, P.Seetharamiah, and K.Soundararajan, "Acoustic sensor network for surveillance, design challenges and solutions", National Symposium on Acoustics, NSA 2010.

[9] Tanenabum Andrew S, Computer Networks, Pearson Education, 2003.

[10] Vijay Chandrasekhar, Wiston KG Seah, Yoo Sag Choo, How Voon Ee, "Localization in Underwater Sensor Networks- Survey and Challenges", WUWNet'06, September 2006, LosAngeles, USA.

[11] S.V.Rangarajan, Abraham Varughese, P.Seetharamiah, K.Soundararajan, "Localisation Schemes in an Underwater Sensor Network - Survey and Solutions" International Conference on Sensors \& Related Networks, SENNET'2012

[12] Hai Yan, Zhijie Shi and Jun-Hong Cui, DBR: DepthBased Routing for Underwater Sensor Networks, in Proc. IFIP Networking, pp. 1--13, May 2008.

[13] S.V.Rangarajan, Abraham Varughese, P.Seetharamaiah, K.Soundararajan, "UNET - A Network simulator for underater Networks" International journal of Computer Applications, Volume 50 - No.15, July 2012, Newyork, USA 